\title{
Quantitative Measurement of Scientific Software Quality: Definition of a Novel Quality Model
}

\author{
Bojana Koteska* and Anastas Mishev ${ }^{\dagger}$ \\ Faculty of Computer Science and Engineering \\ Rugjer Boskovikj 16, 1000 Skopje, Macedonia \\ *bojana.koteska@finki.ukim.mk \\ †anastas.mishev@finki.ukim.mk \\ Ljupco Pejov \\ Faculty of Natural Sciences and Mathematics \\ Arhimedova 5, 1000 Skopje, Macedonia \\ ljupcop@pmf.ukim.mk
}

Received 16 August 2017

Revised 9 October 2017

Accepted 17 November 2017

\begin{abstract}
This paper presents a novel quality model, which provides a quantitative assessment of the attributes evaluated at each stage of development of scientific applications. This model is defined by selecting a set of attributes and metrics that affect the quality of applications. It is based on the established quality standards. The practical application and verification of the quality model is confirmed by two case studies. The first is an application for solving onedimensional and two-dimensional Schrödinger equations, using the discrete variables representation method. The second is an application for calculating an ECG-derived heart rate and respiratory rate. The first application follows a development model for scientific applications, which includes some software engineering practices. The second application does not use a specific development model, rather, it is developed ad hoc. The quality of the applications is evaluated through comparative analyses using the proposed model. Based on software quality metrics, the results of this study indicate that the application for solving one-dimensional and two-dimensional Schrödinger equations produces more desirable results.
\end{abstract}

Keywords: Scientific software; quality model; quantitative evaluation; quality attributes; metrics.

\section{Introduction}

Every physical object with known characteristics can be modeled and simulated through the development of scientific applications. Scientific applications are

This is an Open Access article published by World Scientific Publishing Company. It is distributed under the terms of the Creative Commons Attribution 4.0 (CC-BY) License. Further distribution of this work is permitted, provided the original work is properly cited. 
software applications that simulate the activities and properties of real-world objects by turning them into mathematical models [1]. Scientific applications solve problems in various fields, such as computational chemistry and physics, bioinformatics, biology, mathematics, etc.

Scientific applications are usually developed by scientists who work in small teams and learn programming languages on their own without any formal training [2]. It is crucial for scientists to obtain accurate results, but they are not concerned with the optimization and parallelization of code. They are guided by the impetus to prove the hypothesis once. If this occurs, scientists are satisfied, and determine that there is no need to make code improvements [3]. Scientists focus primarily on the importance of the algorithm's quality rather than its creation [4]. Scientific application development starts with the coding phase. Scientists believe that most requirements do not need to be written, only discussed [5]. They do not record any formal requirements. So, when software testing is performed, there is no written information about which requirements are completed and which are not $[5,6]$. Scientists value software according to its progress in scientific research, so validation is not critical until an error affects the accuracy of results [6].

The reason for the inferior quality of many applications is a lack of establishing and adhering to formal methods and software engineering practices [4, 6-8]. As a result, more errors are found, the code is difficult for other scientists to read and understand, unnecessary resources are used, and there are issues with refactoring and optimization [9].

If researchers and developers use standardized software engineering practices, the quality of scientific applications can be improved [2, 10-12]. In [10], the authors emphasize use-cases, design patterns and systems architecture as important and useful methods during scientific software development. Kelly and Sanders [11] recommend the following software development approaches, defined by the software engineering community: dynamic testing, static inspection, and formal methods. According to [12], the development of scientific applications requires good project management, risk identification, software quality engineering, validation, and verification. Hernández et al. [13] describe an approach to simplify the development of scientific applications by designing tools, applying domain engineering concepts, and using domain-specific modeling, which are modern software engineering methods for automating software development.

The motivation to conduct this research comes from a desire to prove formally that adopting software engineering practices can improve the quality of scientific applications. Even if the scientific application code produces accurate results, problems with quality can still occur, such as performance, late software requirements, or changes to the development environment, etc. This paper presents a novel model designed for measuring the quality of scientific applications. The main scientific contribution of this research is a model that provides quantitative measurement of the quality attributes of scientific applications, which helps researchers compare the quality results among different scientific applications. Metrics and attributes 
proposed in the quality standards were researched, and will be valuable for measuring the quality of scientific applications.

The remaining sections are organized as follows. Section 2 presents the related work. Section 3 describes the quality model in terms of quality attributes, metrics, and their formal definitions. Section 4 shows the practical application of the proposed quality model by making comparative analysis of quality evaluation results between two different scientific applications. The conclusion and future work are given in the last section.

\section{Related Work}

Scientific software quality models have been discussed in several publications.

In [14], the authors proposed a model for checking scientific software while overcoming the drawbacks of traditional models, such as testing of a given software by considering only critical modules and leaving out noncritical modules. This model fulfills the requirements of the IEEE 12207 standard.

A literature review of 19 open-source software quality assessment models is presented in [15]. The classification of the models is done by their quality characteristics, the methodology they use for assessment, and their domain of applications. Quality models for computation-dominant software as scientific software are also covered.

In his book [16], Alexander provided a list of quality metrics valuable for scientific software. Most of the proposed metrics measure quantitative statements about the code. The other metrics offer some facts about the software and its dynamics.

In [17], the authors provided a probabilistic approach for computing high-level software quality characteristics by aggregating low-level attributes to higher levels. They applied the model to two software systems implemented in the Java programming language, showing that the model correlates with how experts define quality.

In [18], the authors proposed a pragmatic software quality model that could be used for assessing software operating in certain environments. Their model consists of four main components: behavioral attributes, impact attributes, responsibility and measurement of metrics, and classification of attributes and weight factors. The Weighted Scoring Method applied in this model is beneficial and valuable to stakeholders who are more interested in the overall quality, and therefore, assigning weights to reflect business requirements.

Both models provide quantitative assessment of the quality attributes. However, they are not suitable for assessing the quality of scientific applications. These models include metrics related to the requirements of clients and businesses, but are not important in scientific software development.

A model for evaluating software quality in e-Book, which represents an extension of the ISO 9126 model for assessment, is presented in [19]. The research emphasized important quality attributes, but did not provide a quantitative approach. 
Lochmann and Goeb [20] presented a general quality model, which can be used to integrate the various concepts found in standards, quality models, guidelines, and static code checker rules. However, this model does not include specific metrics for quantification of software quality. Other researchers made comparative studies of software quality models by comparing their characteristics and relationships $[21,22]$.

\section{Scientific Software Quality Model}

\subsection{Model definition}

The scientific software quality model is defined by selecting a set of attributes that affect the quality of scientific applications. The attributes selected are those determined to be the most important success factors in scientific application development. Unlike the commercial application development, where team management, profit, client satisfaction, and software correctness have equal roles, the main emphasis in scientific application development is on the correctness of the software.

The purpose of the quality model is to assess the quality of scientific software through certain metrics that measure the software attributes included in the model.

According to the quality standards IEEE Std. 1061 [23] and ISO Std. 9126 [24], there is no universal set of attributes that determine the quality of the software. The selection of the attributes depends on the type of problem and the circumstances under which the software is being developed.

The model for evaluating the quality of scientific applications includes quality attributes that can be further broken down into other attributes. At the lowest level, the model includes metrics that can quantitatively express the selected attributes. More specifically, at the highest level (first level), the model contains the attribute "software quality". Next, the second level consists of the attributes important for measuring the software quality of scientific applications. The attributes of the second level are selected because of their appropriateness in specifying quality objectives for scientific applications. These attributes can be then broken down into other attributes until specific metrics (quantifiable measurements) are defined. The lowest level contains only metrics.

Formally, the dependence among attributes of higher and lower levels can be represented as follows: let $\mathrm{QA}=\left\{A_{1}, A_{2}, \ldots, A_{n}\right\}$ denotes the set of higher level quality attributes. Each attribute $A_{i}$ from the set QA can be broken down into other attributes that belong to the set of lower level attributes for the attribute $A_{i}$, $\mathrm{QA}_{i}=\left\{A_{i_{1}}, \ldots, A_{i_{m}}\right\}$, where $m$ is an integer number.

The model provides an opportunity to select the appropriate attributes important for measuring the scientific software quality. Additionally, the attributes can be broken down into different metrics that are significant for measuring those attributes. Each metric or attribute can be weighted differently. 


\subsection{Selection of quality attributes and metrics}

Considering the characteristics of scientific applications, the quality model for scientific applications contains the following set of second level attributes: $\mathrm{QA}=\{M, P, R\}$, where $M$ refers to maintainability, $P$ denotes portability, and $R$ represents reliability. The definitions of these attributes according to the IEEE Std. 1061 are given below:

- Maintainability - refers to the effort required for performing specific changes to the software. Changes may include bug corrections, software improvements, or adaptation to achieve compliance with the requirements or execution environment.

- Portability — the ability of the software to be transferred from one environment to another.

- Reliability - the ability of the software to maintain the level of performance under stated conditions for a specified period.

Why were these attributes chosen? The selection of these attributes is based on the characteristics of scientific applications. The main purpose of a scientific application is to efficiently handle large datasets and to calculate the results within the allowed time and resource limit.

Regarding maintainability, scientific applications should be structured to provide an opportunity for change without enormous effort. Maintainability becomes a critical factor when bugs must be fixed; the code should be well-structured so that the programmer knows exactly which software parts are affected when a software error occurs. Sometimes, changes may be necessary to increase the efficiency of an application because of limited memory resources and time.

Portability is an important aspect of scientific software quality, as certain parts of an application may be reused. Occasionally, an entire software solution may be reused by adding new features, especially when an application is developed in a similar scientific domain. The most crucial factor for increasing the portability is independence of the software modules.

Reliability is critical for scientific applications because simulations may take a long time, and the software must remain in a stable condition and be error-free as long as possible.

According to the IEEE Std. 1061, there are also other important quality attributes, such as functionality, efficiency, and usability. However, these attributes are not included in the quality model for scientific applications because these attributes are associated with users and, in scientific applications, the end-users are usually the scientists themselves. Functionality confirms the specific properties and functions to meet user requirements. Usability determines the user effort required to use the software. Efficiency includes utilization of the human factor in the development process but, because scientific applications are usually developed by one scientist, this factor does not affect the quality of a scientific application. 
The quality attributes from the set of second level attributes $\mathrm{QA}=\{M, P, R\}$ can be further broken down into other attributes or into directly measurable metrics. In addition, the metrics that measure a certain attribute can be weighted differently, depending on the requirements of the software system.

Figure 1 shows the selection of attributes and metrics for evaluating scientific software quality. The yellow rectangles represent the attributes and the blue rectangles represent metrics.

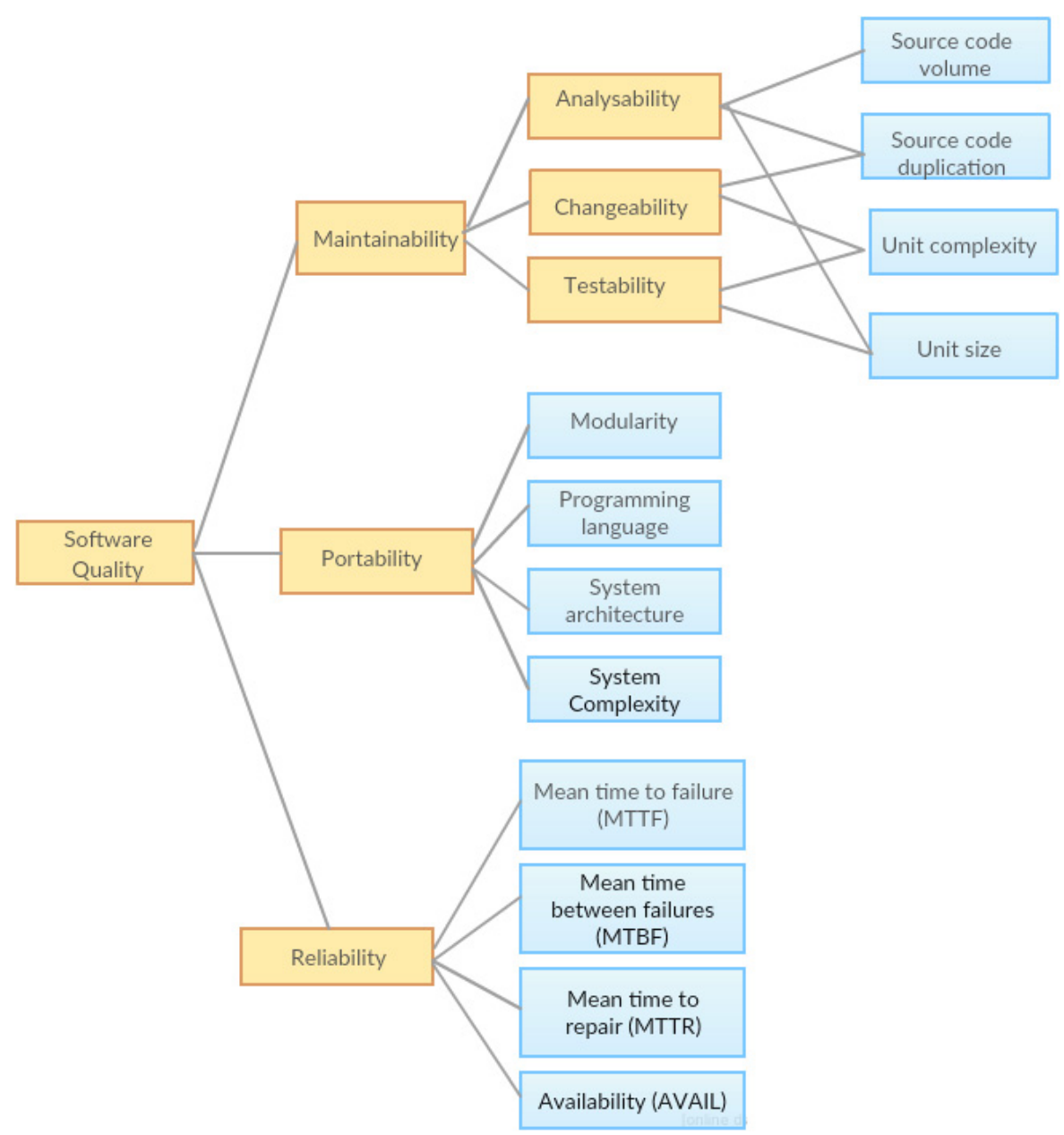

Fig. 1. Attributes and metrics determining the scientific software quality.

\subsection{Calculating the maintainability attribute}

Maintainability $(M)$ depends on the attributes: analyzability, changeability, and testability [24]. According to the ISO 9126-1 standard, analyzability is defined as 
the ability of the software product to be diagnosed with deficiencies or causes of errors, and to identify the parts that need to be modified. Changeability is the ability of the software product to enable a modification. Testability is the ability of the software product to enable modified software to be validated.

In the proposed quality model, the following two scenarios were explored to determine the maintainability of scientific software:

(1) Software maintainability can be calculated directly by calculating the Maintainability index metric [25].

(2) Software maintainability can be calculated by defining attributes that determine maintainability (i.e. analyzability, changeability, and testability) and metrics for measuring these attributes [26].

\subsubsection{Scenario 1}

The Maintainability index metric is defined as follows:

$$
\mathrm{MI}=171-5.2 * \ln (V)-0.23 *(G)-16.2 * \ln (\mathrm{LOC}),
$$

where $V$ is Halstead Volume [27], $G$ denotes cyclomatic complexity [28] and LOC is the count of source lines of code.

According to Halstead, the volume of a source code is calculated as follows:

$$
V=N * \log _{2}(n)
$$

where $N$ is the total number of operations and operands and $n$ is the number of different operations and operands found in the source code.

The cyclomatic complexity refers to the control flow graph generated from the source code. It is used to define the minimum number of module test cases. It is calculated as follows:

$$
G=E-N+P
$$

where $E$ is the number of edges in the graph, $N$ is the number of vertices, and $P$ is the number of graph connected components. If the graph is connected, then $P=2$. A simplified variant of this formula is $G=D+1$, where $D$ denotes the number of decision points in the graph.

The derived formula for calculating the Maintainability index designed by Microsoft Visual Studio (version 2008) uses a scale of 0-100 and it has the following form:

$$
\mathrm{MI}=\operatorname{MAX}(0,(171-5.2 * \ln (V)-0.23 *(G)-16.2 * \ln (\mathrm{LOC})) * 100 / 171) .
$$

The Maintainability index formula defined by the Institute of Software Engineering has the following form:

$$
\mathrm{MI}=171-5.2 * \log _{2}(V)-0.23 * G-16.2 * \log _{2}(\mathrm{LOC}) .
$$


According to the formula implemented in Visual Studio, if the Maintainability index is less than 10, software maintainability is low, and if the Maintainability index is greater than 20, the maintainability is acceptable. According to Coleman et al. [29], the boundaries are 65 and 85, instead of 10 and 20 .

\subsubsection{Scenario 2}

In this scenario, software maintainability is broken down into three attributes: analyzability, changeability, and testability. Analyzability is measured by three metrics: source code volume, source code duplication, and unit size. Changeability is measured by source code duplication and unit complexity metrics. Testability is measured by unit complexity and unit size metrics.

- Source code volume negatively affects the software analyzability. When different programming languages are used, a normalization of the language productivity can be made [30]. An example of the source code volume metric is the number of LOC with the following rules applied: all the blank lines and comment lines are removed, and all the lines with less than two characters are removed. Another option is to calculate man year (MY) and thus to determine the category of the software. The calculation can be done according to the programming languages productivity table [30]. This table displays how many lines of source code (LOC) correspond to function points (FPs), and how many FPs a programmer can produce monthly using a specific programming language. Accordingly, if the number of FPs obtained from the code lines is divided by the number of FPs per programmer multiplied by 12 (for a year), the result will be an MY. One possible division of categories would be the following: 0-8 MY, 8-30 MY, 30-80 MY, 80-160 MY, > $160 \mathrm{MY}$, where $0-8 \mathrm{MY}$ is the best result and $>160 \mathrm{MY}$ is the worst result [26].

- The complexity of source code logical units adversely affects the changeability and analyzability of the software system. The complexity of the software units can be calculated using Eq. (3) defined in the Scenario 1 above. According to the Institute of Software Engineering [31], there are four risk categories for cyclomatic complexity of software units: (1-10) — simple, without much risk; (11-20) - a little complex, moderate risk; (21-50) complex, high risk, and (>50) — without the possibility of testing, very high risk. Using this classification, one can determine the percentage of LOC for each category.

- The degree of source code duplication (source code cloning) adversely affects the analyzability and changeability of the software. All the blocks of code with at least six repeated code lines are considered as duplicated codes. The degree of source code duplication can be calculated as a percentage of duplicated LOC. If this percentage is less than $10 \%$, then the code is acceptable [32, 33].

- The size of the logical units of software negatively affects analyzability and testability of the software system and, ultimately, the testability of the entire 
code. The metric for measuring the size of logical units is the number of LOC $[26,34]$.

\subsection{Calculating the portability attribute}

Portability of software can be measured by the following metrics: modularity (Mo), programming language $(\mathrm{Pl})$, assessment of the system architecture $(\mathrm{Oa})$ and the complexity of the system (Co) [35].

The system modularity metric is represented by the following formula:

$$
\mathrm{Mo}=\mathrm{Mn} * 0.5+1 / \mathrm{Ms} * 0.5,
$$

where $\mathrm{Mn}$ is the number of modules in the system and Ms is the average module size (expressed in LOC) [25, 35].

The assessment of the system architecture metric $\mathrm{Oa}$ is calculated as follows: if an independent platform programming language is used, then the resulting value is 1 . If not, the value is obtained from the following three parameters: how much the platform-dependent code is separated from the platform-independent code, usage of standardized and widely available application program interfaces (APIs), and standard models for displaying data and usage of platform-dependent libraries. Each parameter represents one-third of the final assessment, and the architecture assessment is valued from 1 to 5 ( 1 means best) [25, 35].

The value for the programming language $(\mathrm{Pl})$ metric is obtained as follows: if the programming language is one of the following languages: Java, C, C++, or Python, the result is 2 . If another high-level programming language is used, the result is $1[25,35]$.

The complexity of the system metric $(\mathrm{Co})$ is calculated according to the following formula [35]:

$$
\mathrm{Co}=G * 0.5+C p * 0.5
$$

where $G$ is the average unit cyclomatic complexity, and $C p$ denotes the average module coupling (degree of interdependence between software modules). The module coupling can be calculated as follows [36]:

$$
C=1-\frac{1}{d_{i}+2 * c_{i}+d_{o}+2 * c_{o}+g_{d}+2 * g_{c}+w+r},
$$

where $d_{i}$ is the number of input data parameters and $c_{i}$ is the number of input control parameters. Accordingly, $d_{o}$ and $c_{o}$ are the numbers for output parameters; $g_{d}$ denotes the number of global variables that are used as data, and $g_{c}$ is the number of global variables that are used for control. For a given module, $W$ is the number of modules called in that module and $r$ is the number of modules from which the module is called. 


\subsection{Calculating the reliability attribute}

According to the American National Standards Institute (ANSI) [37], software reliability is the probability of failure-free software operation for a specified time period in a specified environment. Thus, we can define the metrics that determine the reliability of the system: mean time to failure (MTTF), mean time between failures (MTBF), mean time to repair (MTTR), and availability (AVAIL) [38, 39].

MTTF metric is defined as the average time between two consecutive failures of the software system [40].

MTTR metric is defined as the average time needed to repair the system after a failure [41].

MTBF is defined as [38]

$$
\mathrm{MTBF}=\mathrm{MTTF}+\mathrm{MTTR} .
$$

The AVAIL metrics determines the reliability of the system and can be calculated as

$$
\mathrm{AVAIL}=\frac{\mathrm{MTBF}}{\mathrm{MTBF}+\mathrm{MTTR}}
$$

\section{Using the Quality Model in Practice}

This section shows the practical application of the proposed quality model for evaluating the quality of scientific software applications.

\subsection{Methodology}

The evaluation of the quality model was done by quantification of the quality attributes of two different scientific applications. The quality metrics presented in Fig. 1 (blue rectangles) were calculated for both applications. Then, the results for the metrics and attributes were compared.

As a part of our previous research, we developed two scientific applications:

(1) Application for solving one-dimensional and two-dimensional Schrödinger equations (A1) [42].

(2) Application for calculating ECG-derived heart rate and respiratory rate (A2) [43].

Both applications are written in the C programming language. A1 has 1074 LOC organized into 48 logical units. A1 was developed according to the proposed model for the development of scientific applications described in our previous work [44]. This model adapts software engineering practices to the development process of scientific applications. The proposed development model is a combination of the incremental software development model, which combines the waterfall and iterative development model, and the prototype model. The combination of these two 
software development models means that software is developed in increments, but each increment is a complete elaboration of a single subsystem or function (vertical prototype). The reason for choosing this combination of incremental and prototyping software development models is the nature of scientific software. Scientific software is complex simulation software developed and running on a high-performance computer [45]. Our goal is to define a scientific software development model, which will allow development of software in small, independent units. Additionally, each of these units needs to be an independent subsystem that can be reused in other scientific applications from the same or similar scientific domain.

Furthermore, test case design is added before the implementation phase in each increment. Each increment contains eight phases: Requirements Analysis, System Design, Test Cases Design, Implementation, Testing and Evaluation. The proposed development model for scientific software consists of increments followed by integration testing, which is testing of individual software modules as a group, and deployment of the whole system. The use of this development model results in specified requirements, modular code, specified test cases, automated tests, and generated documents.

A2 has 1148 LOC organized into 26 logical units. A2 is developed ad hoc, starting directly from the programming phase. No software engineering practices are used during the development process.

These applications were chosen due to their ability to prove that the developers who adopt software engineering practices while creating applications (A1) produce better results for all measured quality metrics than developers who create software applications ad hoc (A2).

The data required for the practical evaluation of the model are obtained by analyzing the software applications' source codes and the software applications performance analysis. To measure the source code metrics, tools are used to inspect the source code. Details about the software tools used are provided in the next subsections. Some of the metrics were measured manually.

Data analysis was performed by analyzing the results obtained for the same quality metrics for A1 and A2. The metrics results are used for quantification of the quality attributes. They are weighted equally. For example, the changeability attribute is broken down into two metrics: source code duplication and unit complexity. The changeability attribute value is an average of both metric values. If some of the metrics affect the attribute negatively, then inverse values of the metrics are taken into consideration. Since both metrics inversely affect the changeability, it indicates that the application with the lower changeability value is more capable of change.

\subsection{Evaluation of the maintainability attribute}

The calculation of software maintainability is made according to the scenarios defined in Sec. 3.3. To calculate the software volume according to Halstead $(V)$, 
the Halstead Metrics Tool is used [46]. The results of calculating the Maintainability index MI for both applications are shown in Table 1. G denotes the cyclomatic complexity. LOC is the number of LOC.

Table 1. Results for maintainability index.

\begin{tabular}{ccccc}
\hline & LOC & $V$ & $G$ & MI \\
\hline A1 & 1074 & 27081.28 & 160 & $\mathbf{6 2 . 0 5}$ \\
A2 & 1148 & 30496.80 & 169 & $\mathbf{5 9 . 2 4}$ \\
\hline
\end{tabular}

Although the maintainability results from A1 are favorable, it is not the most relevant factor in measuring the quality of a particular application, since the Maintainability index includes LOC directly as a metric.

According to Scenario 2, software maintainability depends on the following attributes: analyzability, changeability, and testability.

The analyzability attribute is evaluated by using the following metrics:

- Volume - represented in MY. The volume of the source code is calculated according to the tables shown in the programming language tables published by Jones [30]. FPs are calculated when LOC is divided by 128 because both applications use $\mathrm{C}$ as the programming language. Volume in MYs is obtained when the total FPs are divided by the number of FPs monthly per programmer, multiplied by 12 .

- Code duplication - expressed as a percentage of LOC. To detect the percentage of duplicated source code blocks, the tool PMD [47] was used.

- Size of software units - average number of code lines per logical software unit.

The results for the analyzability attribute are shown in Table 2. Since all three metrics inversely affect analyzability, it can be clearly concluded that application A1 had better results. All three metrics are weighted equally.

Table 2. Results for analyzability attribute.

\begin{tabular}{ccccc}
\hline & Volume (MY) & Duplicated code $(\%)$ & Average unit size (LOC) & Analyzability \\
\hline A1 & 0.08 & 5.59 & 16.48 & $\mathbf{7 . 3 8}$ \\
A2 & 0.09 & 17.25 & 44.12 & $\mathbf{2 0 . 4 8}$ \\
\hline
\end{tabular}

Software changeability depends on the percentage of duplicate code and on the average complexity of logical software units. The calculations according to the risk categories for cyclomatic complexity of software units proposed in Sec. 3.3 are shown in Table 3.

The cyclomatic complexity of units for both applications is shown in Fig. 2. It should be noted that application A2 has three modules with cyclomatic complexity greater than 250 , which adversely affects the complexity of the entire application. 
Table 3. Percentage of source code according to the risk categories for cyclomatic complexity.

\begin{tabular}{lrr}
\hline Module complexity & A1 (\%) & A2 (\%) \\
\hline $1-10$ & 27.65 & 20.12 \\
$11-20$ & 25.51 & 2.44 \\
$21-50$ & 4.19 & 9.67 \\
$>50$ & 16.29 & 67.68 \\
\hline
\end{tabular}

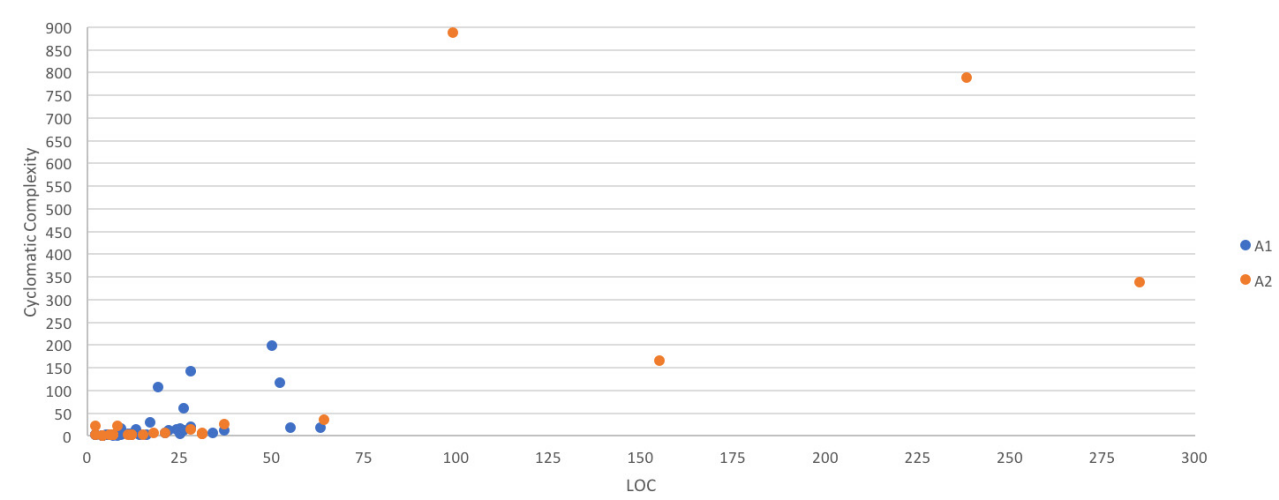

Fig. 2. Cyclomatic complexity of units.

To calculate the software changeability, the average complexity per unit and percentage of duplicated code are used. Both metrics are weighted equally. The results for the changeability attribute are shown in Table 4. Since both metrics inversely affect the changeability, it can be concluded that the application A1 is more capable of change.

Table 4. Results for changeability attribute.

\begin{tabular}{cccc}
\hline & Duplicated code $(\%)$ & Average unit complexity & Changeability \\
\hline A1 & 5.59 & 19.02 & $\mathbf{1 2 . 3 0}$ \\
A2 & 17.25 & 90.77 & $\mathbf{5 4 . 0 1}$ \\
\hline
\end{tabular}

The testability attribute depends on the average unit complexity and the average unit size. Both metrics are weighted equally. The results for the testability attribute are shown in Table 5. Since both metrics inversely affect the software testability, it can be concluded that the software A1 has better results. 
Table 5. Results for the testability attribute.

\begin{tabular}{cccc}
\hline & Average unit size & Average unit complexity & Testability \\
\hline A1 & 16.48 & 19.02 & $\mathbf{1 7 . 7 5}$ \\
A2 & 44.12 & 90.77 & $\mathbf{6 7 . 4 4}$ \\
\hline
\end{tabular}

\subsection{Evaluation of the portability attribute}

The software portability attribute can be calculated using the following four metrics: modularity, programming language, system architecture, and system complexity.

System modularity depends on the number of software units (modules) and the average unit size (LOC); it is calculated using Eq. (6). The number of modules positively affects the modularity of the system, and the average size of modules can negatively affect the system. Both metrics are weighted equally. Modularity results are shown in Table 6. Results show that application A1 is more modular.

Table 6. Results for the modularity attribute.

\begin{tabular}{lccc}
\hline & Average unit size & Number of units & Modularity \\
\hline A1 & 16.48 & 48 & $\mathbf{2 4 . 0 3}$ \\
A2 & 44.12 & 26 & $\mathbf{1 3 . 0 1 1}$ \\
\hline
\end{tabular}

Since both applications use a programming language that is platform-independent, the system architecture value is 1 . The programming language metric value is 2 for both applications.

The complexity of the system is calculated according to Eq. (7). The average unit cyclomatic complexity and the average unit coupling inversely affect the complexity of the system. Modularity results are presented in Table 7, which show that the complexity of application A1 is smaller.

Table 7. Results for the system complexity attribute.

\begin{tabular}{cccc}
\hline & Average unit complexity & Average unit coupling & System complexity \\
\hline A1 & 19.02 & 0.82 & $\mathbf{9 . 9 2}$ \\
A2 & 90.77 & 0.77 & $\mathbf{4 5 . 7 7}$ \\
\hline
\end{tabular}

\subsection{Evaluation of the reliability attribute}

Reliability is calculated using the following metrics: MTTF, MTBF, MTTR, and AVAIL. Only, the MMTR metric inversely affects the reliability.

Metrics are calculated according to the equations presented in Sec. 3.5. Results for the application A1 were measured for an execution time of $173 \mathrm{~min}$, and the results 
Table 8. Results for the reliability attribute.

\begin{tabular}{lcccc}
\hline & MTTF (minutes) & MTTR (minutes) & MTBF (minutes) & AVAIL \\
\hline A1 & 86 & 6 & 92 & 0.94 \\
A2 & 25.37 & 14 & 39.37 & 0.74 \\
\hline
\end{tabular}

for A2 were measured for an execution time of $172 \mathrm{~min}$. The results are presented in Table 8, which shows that application A1 has better results for all metrics.

Figure 3 shows a comparison of the metrics and attributes that determine scientific application quality for A1 and A2. The values of the metrics are scaled from 0 to 1 . In addition, for those metrics or attributes that inversely affect the quality, their reciprocal values are considered. The results show that application A1, which follows the model for the development of scientific applications proposed in our previous work [44], had better values for all metrics that determine the quality of scientific applications.

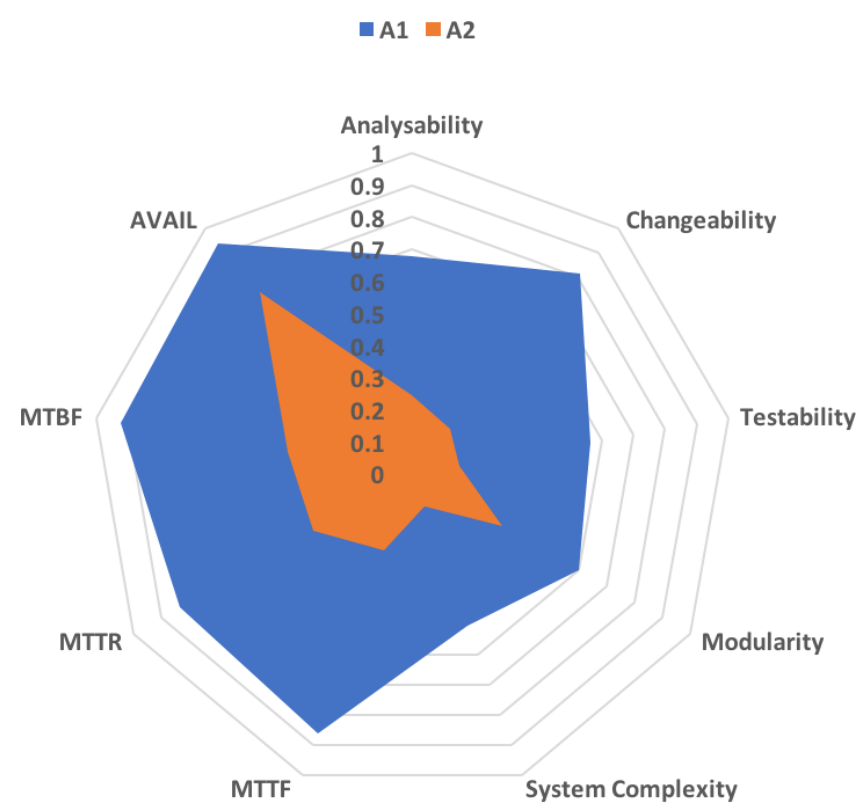

Fig. 3. Comparison of the quality metric results for applications A1 and A2.

\section{Discussion}

The results of the data analysis provide evidence that the proposed scientific software quality model properly assessed the quality of the two applications. Based on the results, the model evaluated the A1 application as the higher-quality application. 
A1 followed some software engineering practices and was developed according to the proposed model for the development of scientific applications described in our paper [44], which is a combination of the incremental software development model and the prototype model. The quality results obtained for A1 can be attributed to several factors. A1 was developed in increments, and each increment represents a single subsystem or function. In each increment, functional and nonfunctional requirements were specified. Test cases were defined for all functional requirements and before the implementation phase. Additionally, the application was evaluated using automated tests and a testing framework. Before deployment, integration testing of the application was done. The source code of the application is organized in smaller modules. In contrast, A2 application was developed ad hoc, starting from the coding phase.

The attributes chosen due to their importance for scientific applications quality are specified as quality attributes in IEEE Std. 1061 and ISO Std. 9126. Our selection of attributes was based on the characteristics of the scientific applications, such as maintainability — scientific applications should be adaptable to changes; portability — scientific applications are often reused in similar scientific domains; reliability — scientific simulations are time consuming. The attributes associated with end-users were eliminated because they are not important factors for the quality of the scientific applications.

The model provides flexibility, in terms of adding new attributes, and metrics that, according to other researchers, are considered as important for measuring the quality of scientific applications. Additionally, the metrics and attributes values can be weighed differently depending on the quality criteria important for the scientific application under evaluation. The model is adaptable to different scientific application types, as it can be expanded to include new quality attributes and metrics. It can also be beneficial for both scientists and software testers.

The proposed quality model requires testing with more complex scientific applications in various scientific fields. If more applications are evaluated using this model, the model can be improved. Additional improvement of the model can be achieved by adding more metrics and attributes as quality criteria for evaluating scientific applications. Furthermore, the model needs to be tested by changing the weight factors of the metric and attributes in different scenarios. Another shortcoming of the model is that most of the metrics for evaluating the scientific software quality require full access to the application's source code. To evaluate the quality of applications that are not open-source, access must be granted to their source codes.

\section{Conclusion and Future Work}

The proposed quality model provides quantitative evaluation of the attributes that are valued at each stage of the development of scientific applications. The model is defined to evaluate scientific applications using the following primary attributes: 
maintainability, portability, and reliability. The model is based on the accepted quality standards.

The practical application of the quality model is shown through two case studies. The first is an application for solving one-dimensional and two-dimensional Schrödinger equations by using the discrete variables representation method. The second is an application for calculating ECG-derived heart rate and respiratory rate. The design of the first application followed the development model for scientific applications proposed in our previous work [44], which includes some software engineering practices. The second application did not use a specific development model. Instead, it was developed ad hoc. The quality of the applications is evaluated through comparative analysis made by using the proposed quality model.

The results showed that the first application had better results for all metrics that determine the quality of scientific software. Future research in this area will be aimed at improving the scientific application quality model by evaluating the quality of scientific applications from different fields with varying complexities.

\section{Acknowledgments}

This work was supported, in part, by the European Unions Horizon 2020 research and innovation programme, project Virtual Research Environment for Regional Interdisciplinary Collaboration in Southeast Europe and Eastern Mediterranean VI-SEEM [675121].

\section{References}

1. I. Ziff Davis Publishing Holdings, 1995, PC Magazine. http://www.pcmag.com/encyclopedia/term/50872/scientific-application.

2. C. Roy, Practical software engineering strategies for scientific computing, in Proc. 19th AIAA Computational Fluid Dynamics Conf., 2009, pp. 1473-1485.

3. R. Baxter, Software engineering is software engineering, in Proc. First Int. Workshop on Software Engineering for High Performance Computing System Application, 2004, pp. $14-18$.

4. V. R. Basili, J. C. Carver, D. Cruzes, L. M. Hochstein, J. K. Hollingsworth, F. Shull and M. V. Zelkowitz, Understanding the high performance computing community: A software engineer's perspective, IEEE Softw. 25(4) (2008) 29-36.

5. J. Segal, Models of scientific software development, in First Int. Workshop on Software Engineering for Computational Science and Engineering, 2008.

6. J. Segal, Scientists and software engineers: A tale of two cultures, in Proc. Psychology of Programming Interest Group, 2008, pp. 44-51.

7. B. Koteska and A. Mishev, Software engineering practices and principles to increase quality of scientific applications, in ICT Innovations 2012, Series Advances in Intelligent Systems and Computing, Vol. 207, eds. S. Markovski and M. Gusev (Springer, Berlin, Heidelberg, 2013), pp. 245-254.

8. G. Wilson et al., Best practices for scientific computing, PLoS Biol. 12(1) (2014) e1001745. 
9. J. Segal, Models of scientific software development, in Proc. 2008 Workshop Software Engineering in Computational Science and Engineering, 2008.

10. P. B. Andersen et al., Software engineering as a part of scientific practice (2011).

11. D. Kelly and R. Sanders, Assessing the quality of scientific software, in First Int. Workshop on Software Engineering for Computational Science \& Engineering, 2016. http://cs.ua.edu/SECSE08/Papers/Kelly.pdf, Last accessed on 10 April, 2016.

12. D. E. Post and R. P. Kendall, Software project management and quality engineering practices for complex, coupled multiphysics, massively parallel computational simulations: Lessons learned from asci, Int. J. High Perform. Comput. Appl. 18(4) (2004) 399-416.

13. F. Hernández, P. Bangalore and K. Reilly, Automating the development of scientific applications using domain-specific modeling, in Proc. Second Int. Workshop on Software Engineering for High Performance Computing System Applications, 2005, pp. $50-54$.

14. G. U. Maheswari and V. R. Prasad, Optimized software quality assurance model for testing scientific software, Int. J. Comput. Appl. 36(7) (2011) 37-46.

15. A. Adewumi, S. Misra, N. Omoregbe, B. Crawford and R. Soto, A systematic literature review of open source software quality assessment models, SpringerPlus $\mathbf{5}(1)$ (2016) 1936.

16. A. Neidhardt, Applied Computer Science for GGOS Observatories: Communication, Coordination and Automation of Future Geodetic Infrastructures (Springer, 2017).

17. T. Bakota, P. Hegedüs, P. Körtvélyesi, R. Ferenc and T. Gyimóthy, A probabilistic software quality model, in 2011 27th IEEE Int. Conf. Software Maintenance, 2011, pp. 243-252.

18. J. H. Yahaya and A. Deraman, Measuring the unmeasurable characteristics of software product quality, Int. J. Adv. Comput. Technol. 2 (2010) 95-106.

19. S. Fahmy, N. Haslinda, W. Roslina and Z. Fariha, Evaluating the quality of software in ebook using the iso 9126 model, Int. J. Control Autom. 5(2) (2012) 115-122.

20. K. Lochmann and A. Goeb, A unifying model for software quality, in Proc. 8th Int. Workshop on Software Quality, 2011, pp. 3-10.

21. S. F. Ahmad, M. R. Beg and M. Haleem, A comparative study of software quality models, Int. J. Sci. Eng. Technol. Res. 2(1) (2013) 172.

22. M. W. Suman and M. Rohtak, A comparative study of software quality models, Int. J. Comput. Sci. Inf. Technol. 5(4) (2014) 5634-5638.

23. E. Iee, IEEE Std 1061-1998, IEEE Standard for a Software Quality Metrics Methodology (1998).

24. ISO/IEC, ISO/IEC 9126. Software Engineering — Product Quality (ISO/IEC, 2001).

25. P. Oman and J. Hagemeister, Metrics for assessing a software system's maintainability, in Proc. Conf. Software Maintenance, 1992, pp. 337-344.

26. I. Heitlager, T. Kuipers and J. Visser, A practical model for measuring maintainability, in 6th Int. Conf. Quality of Information and Communications Technology, 2007, pp. $30-39$.

27. M. H. Halstead, Elements of Software Science (Elsevier, New York, 1977).

28. T. J. McCabe, A complexity measure, IEEE Trans. Softw. Eng. 2(4) (1976) 308-320.

29. D. Coleman, D. Ash, B. Lowther and P. Oman, Using metrics to evaluate software system maintainability, Computer 27(8) (1994) 44-49.

30. C. Jones, Programming Languages Table, Release 8.2 (Software Productivity Research, Burlington, MA, 1996).

31. G. O'Regan, Software engineering institute (sei), in Pillars of Computing (Springer, 2015), pp. 195-205. 
32. K. Mguni and Y. Ayalew, An assessment of maintainability of an aspect-oriented system, ISRN Softw. Eng. 2013 (2013).

33. G. Hegedüs, G. Hrabovszki, D. Hegedüs and I. Siket, Effect of object-oriented refactorings on testability, error proneness and other maintainability attributes, in Proc. 1st Workshop on Testing Object-Oriented Systems, 2010, p. 8.

34. T. Manoharan, Metrics tool for software development life cycle, Int. J. Res. Info. Tech. 2 (2014) 1-16.

35. C. Johnson, R. Patel, D. Radcliffe, P. Lee and J. Nguyen, Establishing qualitative software metrics in department of the navy programs, DTIC Document, Technical Report, 2015.

36. R. S. Pressman, Software Engineering: A Practitioner's Approach (Palgrave Macmillan, 2005).

37. M. H. Saunders, American national standards institute, Ph.D. dissertation, National Institute of Standards and Technology (2017).

38. G. Kaur and K. Bahl, Software reliability, metrics, reliability improvement using agile process, Int. J. Innov. Sci. Eng. Technol. 1(3) (2014) 143-147.

39. K. C. Kapur and M. Pecht, Reliability Engineering (John Wiley \& Sons, 2014).

40. S. Prakash and D. P. Vidyarthi, Maximizing availability for task scheduling in computational grid using genetic algorithm, Concurr. Comput. Pract. Exp. 27(1) (2015) 193-210.

41. E. Michlowicz, Tpm method in the analysis of flow in the cold rolling mill, in Conf. Proc. 22nd Int. Conf. Metallurgy and Materials, 2013, pp. 291-296.

42. B. Koteska, Program for solving 2D Schrödinger equation. https://code.vi-seem.eu/ bojana.koteska/2DSchrodingerEq.

43. B. Koteska and M. Simjanoska, ECG derived vital parameters. https://github.com/ bojanakoteska/ECG-derived-vital-parameters.

44. B. Koteska, L. Pejov and A. Mishev, Framework for developing scientific applications: Solving 1D and 2D schrödinger equation by using discrete variable representation method, in The First Int. Conf. Advances and Trends in Software Engineering, 2015, pp. 93-99.

45. J. Segal and C. Morris, Developing scientific software, IEEE Softw. 25(4) (2008) 18-20.

46. Halstead Metrics Tool, https://sourceforge.net/projects/halsteadmetricstool/.

47. InfoEther, Pmd duplicate code detector. https://pmd.github.io/pmd-5.4.1/usage/ cpd-usage.html. 